\title{
LIMITATION TOPOLOGIES ON FUNCTION SPACES
}

\author{
PHILIP L. BOWERS
}

\begin{abstract}
Four competing definitions for limitation topologies on the set of continuous functions $C(X, Y)$ are compared.
\end{abstract}

\section{INTRODUCTION}

Four competing definitions for limitation topologies on the set $C(X, Y)$ of maps (= continuous functions) from a space $X$ to a space $Y$ are compared. Although all four topologies arise naturally, it is shown that only two of the four topologies are appropriate for describing relationships between elements of $C(X, Y)$ and, in particular, closeness of maps that have been useful in the study of Hilbert space manifolds and other infinite-dimensional nonlocally compact manifolds as influenced by the seminal work of $\mathrm{H}$. Torunczyk.

The limitation topology as originally described by Torunczyk [To2] is a convenient language in which to express the fact that, under specified conditions on $X$ and $Y$, arbitrary maps from $X$ to $Y$ are approximable with cover close control in $Y$ by maps having various desirable properties. For example, if $X$ is a separable metric space and $Y$ is the separable Hilbert space, then maps from $X$ to $Y$ are strongly approximable by embeddings, and this can be stated alternately as the set $\operatorname{Emb}(X, Y)$ of embeddings of $X$ in $Y$ is a dense subset of $C(X, Y)$ whenever $C(X, Y)$ is given the limitation topology. More importantly, the limitation topology has been used as a powerful tool in obtaining quick proofs that certain subsets of $C(X, Y)$ are dense in $C(X, Y)$. These proofs rely on the fact that $C(X, Y)$ becomes a Baire space whenever it is given the limitation topology and this reduces the problem of showing that a subset $G$ is dense in $C(X, Y)$ to observing that $G$ can be written as a countable intersection of open dense subsets. In practice, there is usually a natural decomposition of $G$ as a countable intersection of open subsets and it remains to decide whether each such open set is dense. The equivalence of the following two statements is used both for deciding whether the open sets are dense and for proving that $C(X, Y)$ is a Baire space when given the limitation topology:

$$
E \subset C(X, Y) \text { is dense in } C(X, Y) \text {, }
$$

Received by the editors May 5, 1987 and, in revised form, February 6, 1988.

1980 Mathematics Subject Classification (1985 Revision). Primary 54C35; Secondary 54A10.

Key words and phrases. Limitation topology, modified limitation topology. 
(**) for every $f \in C(X, Y)$ and open cover $\mathscr{U}$ of $Y$ (or, perhaps, im $f$ ), there exists $g \in E \mathscr{U}$-close to $f$.

Any topology on $C(X, Y)$ that implies the equivalence of $(*)$ and $(* *)$ can vie for the title "Limitation Topology." Unfortunately, Torunczyk's original wording of the definition of the limitation topology coupled with the equivalence of $(*)$ and $(* *)$ led many researchers to hold the misconception that the set

$$
\mathscr{S}=\{B(f, \mathscr{U}) \mid f \in C(X, Y), \mathscr{U} \in \operatorname{cov}(Y)\}
$$

(see $\S 2$ for definitions) forms a basis for the limitation topology and/or that each set of the form $B(f, \mathscr{U})$ is open. A secondary goal of this paper is to correct this misconception. Simple examples are constructed that show that this assumption that $\mathscr{S}$ forms a basis for the limitation topology on $C(X, Y)$ in general is invalid. In fact, these examples illustrate that in general $\mathscr{S}$ cannot form a basis for any topology on $C(X, Y)$.

$\S 2$ presents the definitions of the four competing topologies on $C(X, Y)$ and two are shown to be acceptable definitions for limitation topologies in that they imply the equivalence of $(*)$ and $(* *)$. These two are labeled the limitation topology and the modified limitation topology, respectively. The equivalence of all four topologies as suggested by Toruńczyk [To1, comment before (A), p. 33] in case $X$ is compact is demonstrated and other descriptions of the limitation topology in terms of metrics are given.

$\S 3$ presents the aforementioned examples that show that $\mathscr{S}$ in general cannot form a basis for any topology on $C(X, Y)$. Specifically, it is shown that $\mathscr{S}$ cannot form a basis whenever $X$ is not compact and $Y$ is a noncompact absolute retract.

$\S 4$ presents a discussion of subspaces of $C(X, Y)$ and a result of Torunczyk [To2] that implies that $C(X, Y)$ is a Baire space whenever $C(X, Y)$ is given the (modified) limitation topology and $Y$ possesses a complete metric. It is observed that whenever $X$ is homeomorphic to $Y$, all four topologies introduced in $\S 2$ coincide when restricted to the subset of $C(X, Y)$ that consists of homeomorphisms of $X$ onto $Y$.

Terminology and notation. If $f$ and $g$ are maps of a space $X$ into a space $Y$ and $\mathscr{U}$ is a collection of subsets of $Y$, then $g$ is called a $\mathscr{U}$-approximation to $f$ or $g$ is said to be $\mathscr{U}$-close to $f$ provided for every $x$ in $X,\{f(x), g(x)\}$ is contained in some member of $\mathscr{U}$. Given families $\mathscr{A}$ and $\mathscr{B}$ of subsets of a space $Y, \mathscr{A}$ refines $\mathscr{B}$ provided each member of $\mathscr{A}$ is contained in some member of $\mathscr{B}$. Observe that it is not assumed that $\bigcup \mathscr{A}=\bigcup \mathscr{B}$ for $\mathscr{A}$ to refine $\mathscr{B} . \mathscr{A}$ star-refines $\mathscr{B}$ if st $\mathscr{A}=\{\operatorname{st}(A, \mathscr{A}) \mid A \in \mathscr{A}\}$ refines $\mathscr{B}$ where $\operatorname{st}(A, \mathscr{A})$ is the union of all elements of $\mathscr{A}$ that hit $A$, and $\mathscr{A}$ double star-refines $\mathscr{B}$ if $\mathrm{st}^{2} \mathscr{A}=$ st st $\mathscr{A}$ refines $\mathscr{B} . A R(A N R)$ denotes absolute (neighborhood) retract for the class of metrizable spaces. Further terminology and notation is introduced as needed. 


\section{LiMITATION TOPOLOGIES}

For a topological space $Y, \operatorname{cov}(Y)$ denotes the collection of all open covers of $Y$. For a map $f: X \rightarrow Y$ between spaces and a collection $\mathscr{U}$ of subsets of $Y$, the $\mathscr{U}$-neighborhood of $f$ in $C(X, Y)$ is the set $B(f, \mathscr{U})$ that consists of all maps $g: X \rightarrow Y$ that are $\mathscr{U}$-close to $f$. For the map $f, \operatorname{cov}(f) \operatorname{denotes}$ the collection of all open in $Y$ coverings of $\operatorname{im} f$. We present four topologies on $C(X, Y)$ that arise quite naturally.

$\tau$ denotes the topology on $C(X, Y)$ generated by the $\mathscr{U}$-neighborhoods of $f$ as $\mathscr{U}$ ranges over $\operatorname{cov}(Y)$ and $f$ ranges over $C(X, Y)$. The collection $\mathscr{S}=\{B(f, \mathscr{U}) \mid f \in C(X, Y), \mathscr{U} \in \operatorname{cov}(Y)\}$ forms a subbasis of open sets for $\tau$ and $\tau$ is the smallest topology on $C(X, Y)$ in which each $B(f, \mathscr{U}), f \in$ $C(X, Y)$ and $\mathscr{U} \in \operatorname{cov}(Y)$, is open. Closely related to $\tau$ is the finer topology $\tau^{\prime}$ that is generated by the $\mathscr{U}$-neighborhoods of $f$ as $\mathscr{U}$ ranges over $\operatorname{cov}(f)$ and $f$ ranges over $C(X, Y)$, and for which $\mathscr{S}^{\prime}=\{B(f, \mathscr{U}) \mid f \in C(X, Y)$, $\mathscr{U} \in \operatorname{cov}(f)\}$ forms a subbasis of open sets.

Define a collection $\mathscr{T}$ of subsets of $C(X, Y)$ by the rule: a subset $U \subset$ $C(X, Y)$ is an element of $\mathscr{T}$ if for every $f \in U$, there exists $\mathscr{U} \in \operatorname{cov}(Y)$ such that $B(f, \mathscr{U}) \subset U$. If $U$ and $V$ are elements of $\mathscr{T}$ and $B(f, \mathscr{U}) \subset U$ and $B(f, \mathscr{V}) \subset V$ for $\mathscr{U}, \mathscr{V} \in \operatorname{cov}(Y)$, then $B(f, \mathscr{W}) \subset U \cap V$ for any common refinement $\mathscr{W} \in \operatorname{cov}(Y)$ of $\mathscr{U}$ and $\mathscr{V}$. This ensures that $U \cap V$ is an element of $\mathscr{T}$ and obviously implies that $\mathscr{T}$ is a topology on $C(X, Y)$. Closely related to $\mathscr{T}$ is the finer topology $\mathscr{T}^{\prime}$ defined by the rule: $U \subset C(X, Y)$ is an element of $\mathscr{T}^{\prime}$ if for every $f \in U$, there exists $\mathscr{U} \in \operatorname{cov}(f)$ such that $B(f, \mathscr{U}) \subset U$.

Though $\mathscr{S}$ and $\mathscr{S}^{\prime}$ form subbases for the topologies $\tau$ and $\tau^{\prime}$, respectively, examples constructed in $\S 3$ show that in general they do not form bases. Observe that each $\mathscr{T}$-open subset of $C(X, Y)$ is a union of elements of $\mathscr{S}$ but in general not every union of elements of $\mathscr{S}$ is $\mathscr{T}$-open. In particular, the elements of $\mathscr{S}$ themselves are not necessarily $\mathscr{T}$-open. This is demonstrated, for example, by our first example of $\S 3$ where $X=N$ and $Y=\mathbf{R}$. The subset $B\left(g_{+}, \mathscr{U}\right)$ is not $\mathscr{T}$-open since $f \in B\left(g_{+}, \mathscr{U}\right)$ and there is no $\mathscr{W} \in \operatorname{cov}(Y)$ such that $B(f, \mathscr{W}) \subset B\left(g_{+}, \mathscr{U}\right)$. Similarly, each $\mathscr{T}^{\prime}$-open subset is a union of elements of $\mathscr{S}^{\prime}$ but in general not every such union is $\mathscr{T}^{\prime}$-open. The following relationships occur among the four topologies $\tau, \tau^{\prime}, \mathscr{T}$, and $\mathscr{T}^{\prime}$ on $C(X, Y)$ :

$$
\begin{gathered}
\mathscr{T} \subset \mathscr{T}^{\prime} \\
\cap \cap \\
\tau \subset \tau^{\prime} .
\end{gathered}
$$

In general, all the containments are proper; however, Proposition 2.4 and Lemma 2.5 imply that all four topologies are equal to the compact-open topology on $C(X, Y)$ whenever $X$ is compact metrizable and $Y$ is metrizable.

Even though $B(f, \mathscr{U})$ may fail to be $\mathscr{T}$-open, it does have nonempty interior in the topology $\mathscr{T}$ (as proved below) and this is precisely what provides the equivalence of $(*)$ and $(* *)$ from the Introduction whenever $C(X, Y)$ is 
topologized by $\mathscr{T}$. Because of Proposition 2.3 below, we shall call $\mathscr{T}$ the limitation topology on $C(X, Y)$ and $\mathscr{T}^{\prime}$ the modified limitation topology on $C(X, Y)$.

2.1. Lemma. Let $Y$ be a paracompact space, $f \in C(X, Y)$, and $\mathscr{U} \in \operatorname{cov}(f)$. Then $B(f, \mathscr{U})$ has nonempty interior in $\mathscr{T}^{\prime}$ and if $\cup \mathscr{U}=Y$, then $B(f, \mathscr{U})$ has nonempty interior in $\mathscr{T}$.

Proof. Let $Y^{\prime}=\bigcup \mathscr{U}$ and define the derived set of $B(f, \mathscr{U})$, denote $B_{*}(f, \mathscr{U})$, by

$$
B_{*}(f, \mathscr{U})=\left\{g \in B(f, \mathscr{U}) \mid \exists \mathscr{W} \in \operatorname{cov}\left(Y^{\prime}\right) \text { such that } B(g, \mathscr{W}) \subset B(f, \mathscr{U})\right\} .
$$

$B_{*}(f, \mathscr{U})$ is nonempty since it contains the map $f$. Let $g \in B_{*}(f, \mathscr{U})$ and $\mathscr{W} \in \operatorname{cov}\left(Y^{\prime}\right)$ such that $B(g, \mathscr{W}) \subset B(f, \mathscr{U})$. Since $Y$ is paracompact, there exists an open cover $\mathscr{V}$ of $Y^{\prime}$ that star-refines $\mathscr{W}$. If $h \in B(g, \mathscr{V})$, then

$$
B(h, \mathscr{V}) \subset B(g, \text { st } \mathscr{V}) \subset B(g, \mathscr{W}) \subset B(f, \mathscr{U}),
$$

implying that $h \in B_{*}(f, \mathscr{U})$. Hence $B(g, \mathscr{V}) \subset B_{*}(f, \mathscr{U})$ implying that $B_{*}(f, \mathscr{U})$ is $\mathscr{T}^{\prime}$-open and, if $\bigcup \mathscr{U}=Y$, that $B_{*}(f, \mathscr{U})$ is $\mathscr{T}$-open.

2.2. Corollary. Let $Y$ be a paracompact space. The collection $\mathscr{S}_{*}(f)=$ $\left\{B_{*}(f, \mathscr{U}) \mid \mathscr{U} \in \operatorname{cov}(Y)\right\}$ forms a local $\mathscr{T}$-base of $\mathscr{T}$-open sets at $f$ for each $f \in C(X, Y)$ and the collection $\mathscr{S}_{*}=\left\{B_{*}(f, \mathscr{U}) \mid f \in C(X, Y), \mathscr{U} \in \operatorname{cov}(Y)\right\}$ forms a basis for $\mathscr{T}$. The collection $\mathscr{S}_{*}^{\prime}(f)=\left\{B_{*}(f, \mathscr{U}) \mid \mathscr{Q} \in \operatorname{cov}(f)\right\}$ forms a local $\mathscr{T}^{\prime}$-base of $\mathscr{T}^{\prime}$-open sets at $f$ for each $f \in C(X, Y)$ and the collection $\mathscr{S}_{*}^{\prime}=\left\{B_{*}(f, \mathscr{U}) \mid f \in C(X, Y), \mathscr{U} \in \operatorname{cov}(f)\right\}$ forms a basis for $\mathscr{T}^{\prime}$.

2.3. Proposition. Let $Y$ be a paracompact space. If $C(X, Y)$ is topologized by either $\mathscr{T}$ or $\mathscr{T}^{\prime}$ and $E \subset C(X, Y)$, then the following are equivalent:

(*) $E$ is dense in $C(X, Y)$,

(**) for every $f \in C(X, Y)$ and $\mathscr{U}$, where $\mathscr{U} \in \operatorname{cov}(Y)$ if $\mathscr{T}$ is used and $\mathscr{U} \in \operatorname{cov}(f)$ if $\mathscr{T}^{\prime}$ is used, there exists $g \in E \mathscr{U}$-close to $f$.

Proof. (*) implies $(* *)$ is a consequence of the fact that $B(f, \mathscr{U})$ has nonempty interior. $(* *)$ implies $(*)$ is a consequence of the definitions of $\mathscr{T}$-open and $\mathscr{T}^{\prime}$-open sets.

For an example illustrating the failure of 2.3 whenever $C(X, Y)$ is topologized by $\tau$, see $\S 3$.

We now prove that under restrictions on $X$ and $Y$, the four topologies $\tau$, $\tau^{\prime}, \mathscr{T}$, and $\mathscr{T}^{\prime}$ are all equal to the compact-open topology on $C(X, Y)$.

2.4. Proposition (Torunczyk). If $X$ is a compact metrizable space and $Y$ is a metrizable space, then $\mathscr{S}=\mathscr{S}^{\prime}$ forms a basis for the compact-open topology and $\mathscr{S}(f)=\{B(f, \mathscr{U}) \mid \mathscr{U} \in \operatorname{cov}(Y)\}$ forms a local base of open sets at $f$ in the compact-open topology. Furthermore, the four topologies $\tau, \tau, \mathscr{T}$, and $\mathscr{T}^{\prime}$ all coincide with the compact-open topology.

Before proving 2.4 , we prove a preliminary lemma. 
2.5. Lemma. If $X$ is a compact metrizable space, $Y$ is a metrizable space, and $f \in B(g, \mathscr{U}) \in \mathscr{S}$, then there exists a cover $\mathscr{V} \in \operatorname{cov}(Y)$ such that $B(f, \mathscr{V}) \subset$ $B(g, \mathscr{U})$.

Proof. Fix a metric on $Y$ and for each $y \in \operatorname{im} f$ let $\delta(y) \in[0, \infty]$ be the supremum of all $\delta \geq 0$ for which the following holds: for every $x \in f^{-1}(y)$, there exists $U \in \mathscr{U}$ such that $g(x) \in U$ and $N_{\delta}(y) \subset U$, where $N_{\delta}(y)$ denotes the $\delta$-neighborhood of $y$ in $Y$. The compactness of $X$ guarantees that $\delta(y)>$ 0 for all $y \in \operatorname{im} f$ and we claim that there exists $\varepsilon>0$ such that $\varepsilon<\delta(y)$ for every $y \in \operatorname{im} f$. If not, then there exist a sequence $\left\{y_{i}\right\}_{i=1}^{\infty}$ in $\operatorname{im} f$ and $y \in \operatorname{im} f$ such that $y_{i} \rightarrow y$ and $\delta\left(y_{i}\right) \rightarrow 0$. We may assume without loss of generality that $y_{i} \in N_{\delta}(y)$ for each $i$ where $\bar{\delta}=\delta(y) / 2$ and that $\delta\left(y_{i}\right)<\bar{\delta}$ for each $i$. Notice that $N_{\delta}\left(y_{i}\right) \subset N_{\delta(y)}(y)$ for each $i$. From the definition of $\delta$, since $\delta\left(y_{i}\right)<\bar{\delta}$, there exists $x_{i} \in f^{-1}\left(y_{i}\right)$ such that for every $U \in \mathscr{U}$ with $g\left(x_{i}\right) \in U, N_{\delta}\left(y_{i}\right)$ is not contained in $U$. Since $X$ is compact, we may assume by passing to a subsequence if necessary that $x_{i} \rightarrow x$ for some $x \in X$. By continuity, $f(x)=y$ and hence there exists $U \in \mathscr{U}$ such that $g(x) \in U$ and $N_{\delta(y)}(y) \subset U$. Since $g\left(x_{i}\right) \rightarrow g(x)$ and $y_{i} \rightarrow y$, there exists a positive integer $n$ with $\left\{g\left(x_{n}\right), y_{n}\right\} \subset U$. We have $g\left(x_{n}\right) \in U$ and $N_{\delta}\left(y_{n}\right) \subset N_{\delta(y)}(y) \subset U$ which contradicts our definition of $x_{n}$. Hence $\varepsilon$ exists. Let $\mathscr{V}$ be the open cover of $Y$ that consists of the $\varepsilon / 2$-neighborhoods of the points of $Y$. It is a straightforward argument that shows that $B(f, \mathscr{V}) \subset B(g, \mathscr{U})$.

Proof of Proposition 2.4. Observe that $\mathscr{S} \subset \mathscr{S}^{\prime}$. For $B(f, \mathscr{U}) \in \mathscr{S}^{\prime}$, let $\mathscr{U}^{*}=\mathscr{U} \cup\{Y-\operatorname{im} f\}$ and observe that $B(f, \mathscr{U})=B\left(f, \mathscr{U}^{*}\right)$. Since $X$ is compact, $\mathscr{U}^{*} \in \operatorname{cov}(Y)$ and hence $\mathscr{S}^{\prime} \subset \mathscr{S}$. Then $\mathscr{S}=\mathscr{S}^{\prime}$.

In order to show that $\mathscr{S}(f)$ forms a local base of open sets at $f$ in $\tau$, it suffices to show that for finitely many elements, say $B\left(g_{1}, \mathscr{U}_{1}\right), \ldots, B\left(g_{k}, \mathscr{U}_{k}\right)$, of $\mathscr{S}$ each of which contains $f$, there exists $\mathscr{V} \in \operatorname{cov}(Y)$ for which $B(f, \mathscr{V})$ is contained in $\bigcap_{i=1}^{k} B\left(g_{i}, \mathscr{U}_{i}\right)$. For this, use 2.5 to find $\mathscr{V}_{i}$ for $i=1, \ldots, k$ such that $B\left(f, \mathscr{V}_{i}\right) \subset B\left(g_{i}, \mathscr{U}_{i}\right)$ and let $\mathscr{V}$ be a common refinement of $\mathscr{V}_{1}, \ldots, \mathscr{V}_{k}$ covering $Y$. Then $B(f, \mathscr{V}) \subset B\left(g_{i}, \mathscr{V}_{i}\right)$ for $i=1, \ldots, k$.

It is obvious that since $\mathscr{S}(f)$ forms a local basis of open sets at $f$ for each $f$ in $C(X, Y), \mathscr{S}$ forms a basis for $\tau$, and it is easy to show that subsets open in the compact-open topology on $C(X, Y)$ are $\tau$-open (show this for subbasic sets first). For the other direction, if $f \in B(g, \mathscr{U}) \in \mathscr{S}$, then the proof of 2.5 shows that there exists an $\varepsilon>0$ such that $f \in B(f, \varepsilon) \subset B(g, \mathscr{U})$ where $B(f, \varepsilon)=\{h \in C(X, Y) \mid h$ is $\varepsilon$-close to $f\}$ (assuming a fixed metric on $Y$ ). Since $X$ is compact, the compact-open topology on $C(X, Y)$ is metrizable via the sup metric and each $B(f, \varepsilon)$ is open in the compact-open topology. Hence $B(g, \mathscr{U})$ is open in the compact-open topology and thus $\tau$ and the compactopen topology coincide. Since $\mathscr{S}=\mathscr{S}^{\prime}$, we conclude $\tau=\tau^{\prime}$ and since further 2.5 holds, we conclude $\mathscr{T}=\tau$, and finally $\mathscr{T} \subset \mathscr{T}^{\prime} \subset \tau^{\prime}$ implies $\mathscr{T}^{\prime}=\tau$.

If $Y$ is metrizable, then there are other descriptions of the limitation topology. Let $\operatorname{metr}(Y)$ denote the collection of all bounded compatible metrics for 
$Y$. For a metric $\rho \in \operatorname{metr}(Y)$ and map $f \in C(X, Y)$, let $B_{\rho}(f, 1)$ denote the set $\{g \in C(X, Y) \mid \rho(f, g)<1\}$ where $\rho(f, g)$ denotes the supremum of all distances $\rho(f(x), g(x))$ for $x \in X$. It easily follows from [Du, IX, 9.4 , p. 196] that, for each $B(f, \mathscr{U}) \in \mathscr{S}$, there exists a metric $\rho \in \operatorname{metr}(Y)$ such that $B_{\rho}(f, 1) \subset B(f, \mathscr{U})$; conversely, if $\rho \in \operatorname{metr}(Y)$ is given, then $B(f, \mathscr{U}) \subset B_{\rho}(f, 1)$ where $\mathscr{U} \in \operatorname{cov}(Y)$ consists of the open $\rho$-balls in $Y$ of radius $1 / 3$. Therefore an alternative description of the limitation topology whenever $Y$ is metrizable is that a subset $U \in C(X, Y)$ is $\mathscr{T}$-open if and only if, for every $f \in U$, there exists a metric $\rho \in \operatorname{metr}(Y)$ such that $B_{\rho}(f, 1) \subset U$. $E \subset C(X, Y)$ is $\mathscr{T}$-dense if and only if for every $f \in C(X, Y)$ and metric $\rho \in \operatorname{metr}(Y)$, there exists $g \in E$ such that $\rho(f, g)<1$. Furthermore, we have the following result.

2.6 Proposition. Let $Y$ be a metrizable space. The collection $\left\{B_{\rho}(f, 1) \mid f \in\right.$ $C(X, Y), \rho \in \operatorname{metr}(Y)\}$ forms a basis for $\mathscr{T}$.

Proof. We need only show that $B_{\rho}(f, 1)$ is $\mathscr{T}$-open for $f \in C(X, Y)$ and $\rho \in \operatorname{metr}(Y)$. Let $g \in B_{\rho}(f, 1)$ and choose a positive number $\varepsilon<1-\rho(f, g)$ and let $\mathscr{U} \in \operatorname{cov}(Y)$ consist of the open $\rho$-balls in $Y$ of radius $\varepsilon / 3$. Then $B(g, \mathscr{U}) \subset B_{\rho}(f, 1)$.

There is a similar result for the modified limitation topology $\mathscr{T}^{\prime}$. For a map $f \in C(X, Y)$, let $\operatorname{metr}(f)$ denote the collection of all bounded compatible metrics for open subspaces of $Y$ that contain $\operatorname{im} f$; that is, $\operatorname{metr}(f)=$ $\bigcup\{\operatorname{metr}(U) \mid \operatorname{im} f \subset U, U$ is open in $Y\}$.

2.7. Proposition. Let $Y$ be a metrizable space. The collection $\left\{B_{\rho}(f, 1) \mid f \in\right.$ $C(X, Y), \rho \in \operatorname{metr}(f)\}$ forms a basis for $\mathscr{T}^{\prime}$.

The proof of 2.7 is similar to that of 2.6 .

Finally, we mention a result of Torunczyk [To2]. For $\alpha \in C(Y,(0, \infty))$, $\rho \in \operatorname{metr}(Y)$, and $f \in C(X, Y)$, we write $\bar{B}_{\rho}(f, \alpha)=\{g \in C(X, Y) \mid$ $\rho(f(x), g(x)) \leq \alpha(f(x))$ for all $x \in X\}$. Each $\bar{B}_{\rho}(f, \alpha)$ is $\mathscr{T}$-closed and, if $\rho$ is fixed, then $\left\{\bar{B}_{\rho}(f, \alpha) \mid f \in C(X, Y), \alpha \in C(Y,(0, \infty))\right\}$ forms a basis of $\mathscr{T}$-closed subsets of $C(X, Y)$ [To2].

\section{THE EXAMPLeS}

The following simple example illustrates that in general neither $\mathscr{S}$ nor $\mathscr{S}^{\prime}$ can form a basis for any topology on $C(X, Y)$. Let $X$ denote the natural numbers $\mathbf{N}$ with the discrete topology and $Y$ the real numbers $\mathbf{R}$ with the usual euclidean topology. Define maps $f, g_{+}, g_{-} \in C(X, Y)$ by the equations $f(X)=\{0\}, g_{+}(n)=n$ for $n \in X$, and $g_{-}(n)=-n$ for $n \in X$. For each natural number $n$, let $U_{n}$ denote the open interval $(-1 / n, n)$ of $\mathbf{R}$ and $U_{-n}$ the open internal $(-n, 1 / n)$ of $\mathbf{R}$, and let $\mathscr{U}=\left\{U_{i}\right\}$, an open cover of $\bar{Y}^{n}$. Observe that $f \in B\left(g_{+}, \mathscr{U}\right) \cap B\left(g_{-}, \mathscr{U}\right)$. If either $\mathscr{S}$ or $\mathscr{S}^{\prime}$ forms a basis for a topology on $C(X, Y)$, then there is a map $h \in C(X, Y)$ and an open in $Y$ 
covering $\mathscr{W}$ of $\operatorname{im} h$ such that

$$
f \in B(h, \mathscr{W}) \subset B\left(g_{+}, \mathscr{U}\right) \cap B\left(g_{-}, \mathscr{U}\right) .
$$

Since $f \equiv 0$ is $\mathscr{W}$-close to $h$, there exists an element $W$ in $\mathscr{W}$ that contains 0 . Choose $w \in W, w<0$. Define $h^{\prime} \in C(X, Y)$ by

$$
h^{\prime}(n)= \begin{cases}h(n) & \text { if } h(n) \notin W \\ w & \text { if } h(n) \in W .\end{cases}
$$

Observe that $h^{\prime}(n)=w$ for all but finitely many $n$ since the fact that $h$ is $\mathscr{U}$-close to both $g_{+}$and $g_{-}$forces $h(n) \rightarrow 0$ as $n \rightarrow \infty$. Obviously, $h^{\prime} \in B(h, \mathscr{W})$; however, $h^{\prime}$ is not $\mathscr{U}$-close to $g_{+}$, for any such map cannot take on any fixed negative value infinitely often. This contradicts 3.1. Hence, for this particular example, neither $\mathscr{S}$ not $\mathscr{S}^{\prime}$ is a basis for any topology on $C(X, Y)$.

The form of this example can be used to generate many similar examples. In particular, we have the following proposition.

3.2. Proposition. If $X$ is a noncompact metrizable space and $Y$ is a noncompact $A R$, then $\mathscr{S}$ and $\mathscr{S}^{\prime}$ do not form bases for any topologies on $C(X, Y)$.

Proof. Fix a metric on $Y$ and choose a sequence $\left\{x_{n}\right\}_{n=1}^{\infty}$ of $X$ and a double sequence $\left\{y_{n}\right\}_{-\infty}^{\infty}$ of $Y$ (indexed by the integers) of pairwise distinct elements that have no convergent subsequences in $X$ and $Y$, respectively. Choose a double sequence $\left\{\varepsilon_{n}\right\}_{-\infty}^{\infty}$ of positive numbers for which $\varepsilon_{n} \rightarrow 0$ and $\varepsilon_{-n} \rightarrow$ 0 as $n \rightarrow \infty$ and for which $\left\{N_{\varepsilon_{n}}\left(y_{n}\right)\right\}_{-\infty}^{\infty}$ is pairwise disjoint, where $N_{\varepsilon}(y)$ denotes the $\varepsilon$-neighborhood of $y$ in $Y$. For each integer $n \neq 0$, let $U_{n}$ denote $N_{\varepsilon_{n}}\left(y_{0}\right) \cup N_{\varepsilon_{n}}\left(y_{n}\right)$ and let $U_{0}=Y-\left\{y_{n} \mid n \neq 0\right\}$, and let $\mathscr{U}$ denote the open cover of ${ }^{e_{n}} Y$ that consists of the sets $U_{n}$ for all integers $n$. Use the fact that $Y$ is an $A R$ to obtain maps $g_{+}, g_{-} \in C(X, Y)$ that satisfy $g_{+}\left(x_{n}\right)=y_{n}$ and $g_{-}\left(x_{n}\right)=y_{-n}$ for each positive integer $n$. Let $f$ be the constant map $f(X)=\left\{y_{0}\right\}$ and observe that $f$ is $\mathscr{U}$-close to both $g_{+}$and $g_{-}$and, if $h \in C(X, Y)$ is $\mathscr{U}$-close to both $g_{+}$and $g_{-}$, then $h\left(x_{n}\right) \rightarrow y_{0}$ as $n \rightarrow \infty$. If either $\mathscr{S}$ or $\mathscr{S}^{\prime}$ is a basis for a topology on $C(X, Y)$, then there is a map $h \in C(X, Y)$ and an open in $Y$ covering $\mathscr{W}$ of $\operatorname{im} h$ for which

$$
f \in B(h, \mathscr{W}) \subset B\left(g_{+}, \mathscr{U}\right) \cap B\left(g_{-}, \mathscr{U}\right) .
$$

Let $Z=\bigcup \mathscr{W}$. Since $Z$ is an $A N R$, there is a covering $\mathscr{V}$ of $Z$ refining $\mathscr{W}$ such that $\mathscr{V}$-close maps into $Z$ are $\mathscr{W}$-homotopic. Since $f \equiv y_{0}$ is $\mathscr{W}$-close to $h$, there exists $W \in \mathscr{W}$ with $y_{0} \in W$. Hence $y_{0}$ is an element of $Z$ and there exists $V \in \mathscr{V}$ with $y_{0} \in V$. Since $Y$ is a nontrivial $A R$, there is a point $v$ in $V$ different from $y_{0}$. Define a map $h^{\prime \prime}$ on $\left\{x_{n}\right\}_{n=1}^{\infty}$ by

$$
h^{\prime \prime}\left(x_{n}\right)= \begin{cases}h\left(x_{n}\right) & \text { if } h\left(x_{n}\right) \notin V, \\ v & \text { if } h\left(x_{n}\right) \in V\end{cases}
$$

and observe that $h^{\prime \prime}\left(x_{n}\right)=v$ for all but finitely many values of $n$. Since $h^{\prime \prime}$ is $\mathscr{V}$-close and hence $\mathscr{W}$-homotopic to $h \mid\left\{x_{n}\right\}_{n=1}^{\infty}$, the controlled version of the 
Homotopy Extension Theorem implies that $h^{\prime \prime}$ extends to a map $h^{\prime} \in C(X, Y)$ that is $\mathscr{W}$-close to $h$. Thus $h^{\prime}\left(x_{n}\right) \rightarrow v \neq y_{0}$ as $n \rightarrow \infty$. This contradicts our assumption on $h$ and $\mathscr{W}$, thus showing that neither $\mathscr{S}$ nor $\mathscr{S}^{\prime}$ forms a basis for a topology on $C(X, Y)$.

The first example of this section provides a simple-minded example illustrating the failure of 2.3 whenever $C(X, Y)$ is topologized by $\tau$. With $X, Y, g_{+}$, $g_{-}$, and $\mathscr{U}$ as in the first paragraph of this section, let $E$ denote the set of all maps $g$ in $C(X, Y)$ such that im $g$ misses a neighborhood of 0 . It is easy to see that for every $f \in C(X, Y)$ and $\mathscr{V} \in \operatorname{cov}(Y)$, there exists $g \in E \mathscr{V}$-close to $f$. However, $E$ is not $\tau$-dense in $C(X, Y)$ since $E$ has empty intersection with the nonempty $\tau$-open set $B\left(g_{+}, \mathscr{U}\right) \cap B\left(g_{-}, \mathscr{U}\right)$. This example is somewhat artificial and so we offer another example that illustrates the power of the limitation topology $\mathscr{T}$ vis-à-vis the impotence of $\tau$ in the study of the topology of the separable Hilbert space $l_{2}$. For an arbitrary separable metrizable space $X$, any map $f: X \rightarrow l_{2}$ is approximable, with cover close control in $l_{2}$, by $Z$-maps (i.e., maps $f$ such that the closure of $\operatorname{im} f$ is a $Z$-set). This means, precisely, that for each map $f: X \rightarrow l_{2}$ and open cover $\mathscr{U}$ of $l_{2}$, there exists a $Z$-map $g: X \rightarrow l_{2} \mathscr{U}$-close to $f$. This can be stated conveniently in terms of (*) as the set $E$ of $Z$-maps of $X$ into $l_{2}$ is $\mathscr{T}$-dense in $C\left(X, l_{2}\right)$. The proof of this fact goes as follows. Let $\left\{e_{i}\right\}$ be a countable dense set of maps from the Hilbert cube $I^{\infty}$ into $l_{2}$, which exists since $C\left(I^{\infty}, l_{2}\right)$ is separable, and for each $i$ let $E_{i}$ be the set of maps in $C\left(X, l_{2}\right)$ each of whose image misses a neighborhood of the image of $e_{i}$. It is the case that $E$ contains $\bigcap_{i=1}^{\infty} E_{i}$ and since $C(X, Y)$ is a Baire space when topologized by $\mathscr{T}$ (see 4.1), it suffices to show that each $E_{i}$ is $\mathscr{T}$-open and $\mathscr{T}$-dense. It is easy to see that each $E_{i}$ is $\mathscr{T}$-open and since any compact subset of $l_{2}$, in particular, im $e_{i}$, is a (strong) $Z$-set, it follows that (**) holds for each $i$. Hence each $E_{i}$ is $\mathscr{T}$-open and $\mathscr{T}$-dense and we conclude that $(* *)$ holds for $E$. This actually proves something stronger than that $E$ is $\mathscr{T}$-dense, namely, that $E$ contains a $\mathscr{T}$-dense $G_{\delta}$-subset. This result is important in that it can be coupled with the fact that the set of closed embeddings $\overline{\mathrm{Emb}}\left(X, l_{2}\right)$ of a completely metrizable space $X$ into $l_{2}$ is a $\mathscr{T}$-dense $G_{\delta}$-subset of $C(X, Y)$ to obtain the result that the set of closed $Z$-embeddings of such $X$ into $l_{2}$ is $\mathscr{T}$-dense in $C(X, Y)$. This result was used in Toruńczyk's original proof of his topological charcterization of $l_{2}$ [To2].

Contrast the results of the previous paragraph with the situation for the topology $\tau$. It remains true that each $E_{i}$ is $\tau$-open; however, even though (**) holds for each $E_{i}$, we cannot use this to prove that $(* *)$ holds for $E$. The reader may construct particular examples similar to our simple-minded example of the previous paragraph that show that $E_{i}$ is not necessarily $\tau$-dense even though $(* *)$ holds for $E_{i}$. The useful fact that $(* *)$ holds for $E$ (and the more useful fact that $E$ contains a dense $G_{\delta}$-subset in some suitable topology on $\left.C(X, Y)\right)$ is 
not reflected by the use of the topology $\tau$ and cannot be expressed conveniently in the language of $\tau$.

\section{Subspaces AND THe Baire PROPERTY}

Let $F$ be a subset of $C(X, Y)$. It easily is seen that the subset $U$ of $F$ is open in the subspace topology $\mathscr{T}_{F}\left(\mathscr{T}_{F}^{\prime}\right)$ on $F$ determined by $\mathscr{T}\left(\mathscr{T}^{\prime}\right)$ if and only if, for each $f \in U$, there exists $B(f, \mathscr{U}) \in \mathscr{S}\left(\mathscr{S}^{\prime}\right)$ with $B(f, \mathscr{U}) \cap F \subset$ $U$. This follows, for example, from the fact that any $U \subset F$ for which each $f \in U$ has $B(f, \mathscr{U})$ with $B(f, \mathscr{U}) \cap F \subset U$ may be written as

$$
U=F \cap\left[\bigcup\left\{B_{*}(f, \mathscr{U}) \mid B(f, \mathscr{U}) \cap F \subset U\right\}\right] .
$$

Consider the case where $X=Y$ is metrizable and $F$ is the group of autohomeomorphisms of $X$, denoted in the literature by Auth $X$ (Homeo $(X)$ is reserved for denoting Auth $X$ equipped with the compact-open topology). We shall see below that in this case each set of the form $H(f, \mathscr{U})=B(f, \mathscr{U}) \cap$ Auth $X$, where $f \in$ Auth $X$ and $\mathscr{U} \in \operatorname{cov}(X)$, is open in the subspace topology on Auth $X$ determined by $\mathscr{T}$, which equals that determined by $\mathscr{T}^{\prime}$. This implies that the subspace topology on Auth $X$ determined by $\tau$ and $\tau^{\prime}$ both equal that determined by $\mathscr{T}$ and also implies the well-known fact (see [BP, IV, §1]) that the collection $\mathscr{B}=\{H(f, \mathscr{U}) \mid f \in$ Auth $X, \mathscr{U} \in \operatorname{cov}(X)\}$ forms a basis for a topology on Auth $X$, namely the topology $\mathscr{T}_{\text {Auth } X}$, in which the collection $\{H(f, \mathscr{U}) \mid \mathscr{U} \in \operatorname{cov}(X)\}$ forms a basis of open neighborhoods for $f$. This example probably has reinforced the misconception that $\mathscr{S}$ forms a basis for the limitation topology on $C(X, Y)$. To see that $H(f, \mathscr{U})$ is $\mathscr{T}_{\text {Auth } X}$-open where $f \in$ Auth $X$ and $\mathscr{U} \in \operatorname{cov}(X)$, fix a metric $\rho \in \operatorname{metr}(X)$ and a homeomorphism $g \in H(f, \mathscr{U})$ and define for each $x \in X$ the number $\beta(x)$ :

$$
\beta(x)=\sup \left\{\delta>0 \mid \exists U \in \mathscr{U} \text { such that } f(x) \in U \text { and } N_{\delta}(g(x)) \subset U\right\} .
$$

$\beta: X \rightarrow(0, \infty)$ is a lower semicontinuous function and since $0<\beta$, a wellknown result of C. Dowker [Du, VIII, 4.3] provides a continuous $\alpha$ : $X \rightarrow(0, \infty)$ such that $0<\alpha(x)<\beta(x)$ for each $x \in X$. Easily, $\bar{B}_{\rho}\left(g, \alpha \circ g^{-1}\right)$ is contained in $B(f, \mathscr{U})$ forcing $H(g, \mathscr{V})$ to be contained in $H(f, \mathscr{U})$ where $\mathscr{V} \in \operatorname{cov}(X)$ satisfies $B(g, \mathscr{V}) \subset \bar{B}_{\rho}\left(g, \alpha \circ g^{-1}\right)$. It follows that $H(f, \mathscr{U})$ is $\mathscr{T}_{\text {Auth } X}$-open.

We close with an extremely useful and important lemma due to $\mathrm{H}$. Torunczyk. First, a definition. For a subset $F \subset C(X, Y)$ and metric $\rho \in \operatorname{metr}(Y), F_{\rho}$ denotes the $\rho$-closure of $F$; that is, $f \in F_{\rho}$ if and only if $f$ is the $\rho$-uniform limit of maps from $F$.

4.1. Toruńczyk's Lemma (see [To2]). Let $X$ and $Y$ be spaces with $Y$ completely metrizable and topologize $C(X, Y)$ using either the limitation topology $\mathscr{T}$ or the modified limitation topology $\mathscr{T}^{\prime}$. Let $F$ be a subspace of $C(X, Y)$ and $U_{n}$ for each positive integer $n$ an open subset of $C(X, Y)$. If $U_{n} \cap F$ is dense in $F$ for each $n$, then $F$ is in the closure of $\bigcap_{n=1}^{\infty} U_{n} \cap F_{\rho}$, where $\rho$ is any metric in $\operatorname{metr}(Y)$. In particular, $C(X, Y)$ is a Baire space. 
In [To2], Lemma 4.1 with $C(X, Y)$ topologized by $\mathscr{T}$ appears without proof. For completeness, we give a short proof of 4.1 in case $C(X, Y)$ is topologized by $\mathscr{T}^{\prime}$.

Proof. If $\rho$ and $\rho^{\prime}$ are metrics on $Y$, then $\rho+\rho^{\prime}$ is an equivalent metric on $Y$ for which $F_{\rho+\rho^{\prime}} \subset F_{\rho}$. This allows us to assume, without loss of generality, that the metric $\rho \in \operatorname{metr}(Y)$ is complete for if $\rho$ is not complete, we may replace $\rho$ by $\rho+\rho^{\prime}$ for any complete metric $\rho^{\prime} \in \operatorname{metr}(Y)$. We therefore assume that $\rho$ is a complete metric on $Y$.

It suffices to show that if $f \in F$ and $\mathscr{U} \in \operatorname{cov}(f)$, then there exists a map $f^{\prime} \in \bigcap_{n=1}^{\infty} U_{n} \cap F_{\rho}$ that is $\mathscr{U}$-close to $f$. Inductively define a sequence of triples $\left\{\left(f_{i}, \mathscr{A}_{i}, \mathscr{B}_{i}\right)\right\}$ that satisfy the following conditions:

(i) $f_{1} \in U_{1} \cap B_{*}\left(f, \mathscr{B}_{1}\right) \cap F$ where $\mathscr{A}_{1}=\mathscr{B}_{1}=\mathscr{U}$;

(ii) $f_{i} \in U_{i} \cap B_{*}\left(f_{i-1}, \mathscr{B}_{i}\right) \cap F$ where $\mathscr{B}_{i} \in \operatorname{cov}\left(f_{i-1}\right)$ has $\rho$-mesh less than $2^{-i}, \mathscr{B}_{i}$ double-star refines the cover $\mathscr{A}_{i} \in \operatorname{cov}\left(f_{i-1}\right)$ and $\bigcup \mathscr{B}_{i}=\bigcup \mathscr{A}_{i}$ $(i \geq 2)$;

(iii) $\mathscr{A}_{i+1}$ refines $\mathscr{B}_{i}$ and $B\left(f_{i}, \mathscr{A}_{i+1}\right) \subset U_{i} \cap B_{*}\left(f_{i-1}, \mathscr{B}_{i}\right) \quad(i \geq 1$ and where $\left.f_{0}=f\right)$.

The basis step (i) is possible since $U_{1} \cap F$ is dense in $F$ and the derived set $B_{*}\left(f, \mathscr{B}_{1}\right)$ is $\mathscr{T}^{\prime}$-open in $C(X, Y)$ (see the proof of 2.1$)$. For the inductive step, suppose the triples $\left(f_{j}, \mathscr{A}_{j}, \mathscr{B}_{j}\right)$ for $1 \leq j \leq i$ have been chosen satisfying (i), (ii), and (iii). Since $f_{i}$ is a member of the $\mathscr{T}^{\prime}$-open set $U_{i} \cap B_{*}\left(f_{i-1}, \mathscr{B}_{i}\right)$, there exists a cover $\mathscr{A}_{i+1} \in \operatorname{cov}\left(f_{i}\right)$ refining $\mathscr{B}_{i}$ such that (iii) holds. Let $\mathscr{B}_{i+1}$ be a double star-refinement of $\mathscr{A}_{i+1}$ by open sets in $Y$ covering $\bigcup \mathscr{A}_{i+1}$ and of $\rho$-mesh less than $2^{-(i+1)}$. Since $U_{i+1} \cap F$ is dense in $F$ and the derived set $B_{*}\left(f_{i}, \mathscr{B}_{i+1}\right)$ is $\mathscr{g}^{\prime}$-open, we may choose $f_{i+1}$ so that (ii) holds for $f_{i+1}$.

By (ii) and our choice of the covers $\mathscr{B}_{i}$, it is clear that the sequence $\left\{f_{i}\right\}$ is a $\rho$-Cauchy sequence of maps. Since $\rho$ is a complete metric, the sequence $\left\{f_{i}\right\}$ coverges uniformly with respect to $\rho$ to a map $f^{\prime} \in C(X, Y)$. Hence $f^{\prime} \in F_{\rho}$ and it remains to show that $f^{\prime} \in U_{i}$ for each $i$ and that $f^{\prime}$ is $\mathscr{U}$-close to $f$. First, use (ii) to show that given $k \geq i, f_{k}$ is st $\mathscr{B}_{i+1}$-close to $f_{i}$. This forces $f^{\prime}$ to be st $^{2} \mathscr{B}_{i+1}$-close to $f_{i}$ and hence $\mathscr{A}_{i+1}$-close to $f_{i}$. (iii) now implies that $f^{\prime} \in U_{i}$. Finally, since $f^{\prime}$ is $\mathscr{A}_{2}$-close to $f_{1}$, (iii) again implies that $f^{\prime}$ is $\mathscr{B}_{1}$-close to $f_{0}=f$. Since $\mathscr{B}_{1}=\mathscr{U}$, we are done.

Addendum. For another point of view on topologies on $C(X, Y)$, see [Mc1]. The limitation topology on $C(X, Y)$ is the fine uniform topology of [Mc1]. Proposition 2.6 of this paper is the same as [Mc1, Proposition 1.1] and a proof that $C(X, Y)$ with the limitation topology is a Baire space if $Y$ is completely metrizable appears in McCoy's paper [Mc1, Theorem 1.4]. Some fairly general conditions are given in [Mcl] that imply that certain topologies on $C(X, Y)$ are exactly the compact-open topology. See in particular [Mcl, Proposition 1.2]. In another paper by R. A. McCoy [Mc2], a study is made of the convergence 
of sequences in the topology $\tau$ on $C(X, Y)$. In that paper, $\tau$ is labeled the open-cover topology.

\section{REFERENCES}

[BP] C. Bessaga and A. Pelczynski, Selected topics in infinite dimensional topology, PWN, Warsaw, 1975.

[Du] J. Dugundji, Topology, Allyn and Bacon, Boston, Mass., 1966.

[Mc1] R. A. McCoy, Fine topology on function spaces, Internat. J. Math. Math. Sci. 9 (1986), 417427.

[Mc2] _ , The open-cover topology on function spaces, Fund. Math. 104 (1979), 69-73.

[To1] H. Torunczyk, On CE-images of the Hilbert cube and characterization of Q-manifolds, Fund. Math. 106 (1980), 31-40.

[To2] __, Characterizing Hilbert space topology, Fund. Math. 111 (1981), 247-262.

Department of Mathematics, The Florida State University, Tallahassee, Florida 32306 